\title{
Measurements of Effective Refractive Index of SOI Waveguides using Interferometers
}

\author{
Sarvagya Dwivedi ${ }^{1}$, T. Van Vaerenbergh ${ }^{1,3}$, Alfonso Ruocco ${ }^{1}$, Thijs Spuesens ${ }^{1}$, Peter \\ Bienstman $^{1}$, Pieter Dumon ${ }^{1,2}$ and Wim Bogaerts ${ }^{1,2}$ \\ ${ }^{1}$ Photonics Research Group, Department of Information Technology Ghent University - imec, Center for Nano- and \\ Biophotonics, Sint-Pietersnieuwstraat 41, B-9000 Ghent, Belgium. \\ 2 Also with: Luceda Photonics, Noordlaan 21, 9200 Dendermonde, Belgium \\ ${ }^{3}$ Now with: HP Labs, 1501 Page Mill Road, Palo Alto, CA 94304, USA. \\ sarvagya.dwivedi@intec.ugent.be
}

\begin{abstract}
We demonstrate an accurate method of measuring the effective refractive index of SOI waveguides in the C-band using three Mach-Zehnder Interferometers. Over wafer the average extraction error of effective index and group index is 0.003 and 0.004 .
\end{abstract}

OCIS codes: (230.7370) Waveguides, (230.7408) Wavelength filtering devices

\section{Introduction}

Silicon is a high index contrast material and due to this it is possible to make micron size devices on chip. However, the exact value of the effective refractive index $\left(n_{e f f}\right)$ is critical for the performance of wavelength filters [1]. Given the extreme sensitivity of a silicon wire waveguide to the exact geometry, it is important to measure the effective index in situ, without perturbing the actual waveguide geometry. Existing methods to extract the dispersion such as prism coupling are either not suited for high refractive index waveguides, or actively perturb the waveguide [2]. Non-invasive methods such as ellipsometry cannot be easily applied to narrow waveguides. Techniques using scattering with weak higher-order periodic perturbations [3] actively modify the waveguide, but also change the local pattern density, and thus could even influence the fabrication process. Methods which rely on features in the tranmission spectrum of interferometric devices will measure the group index $n_{g}$ rather than the effective index $n_{e f f}$ [4]. Because silicon wire waveguides are very dispersive, $n_{g}$ and $n_{\text {eff }}$ are very different, and highly wavelength dependent. Therefore, a method to extract both values unambiguously, and with their wavelength dispersion, is needed. We present a simple approach to measure accurately the $n_{\text {eff }}$ of a silicon photonics wire. This method uses three Mach-Zehnder interferometers (MZIs) designed with the same photonic wire and located adjacent to each other. MZIs are the best candidates due to being simple in design, linear in phase response and flexible in choosing the filter order. Other methods of parameter extraction using ring resonators [5] suffer from nonlinearity in terms of phase and higher input optical power due to their resonant nature. It also averages the $n_{e f f}$ over bends and straight sections.In our method two MZIs have a low order $m$, i.e. a large free spectral range (FSR) which allows us to estimate the order of the filter correctly within the C-band.The third one, of much higher order, has a small FSR to extract the wavelength dependence of the group index $\left(n_{g}\right)$. Wafer-scale measurements are also performed and presented.

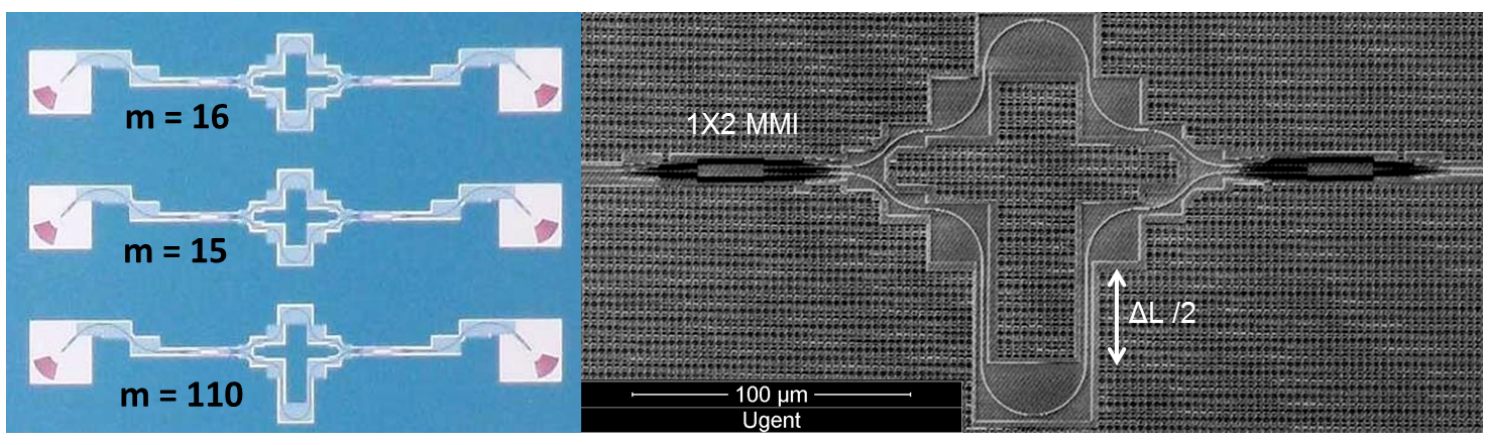

Fig. 1: (a). Camera image of fabricated devices and (b) SEM image of fabricated MZI with $\mathrm{m}=110$. 


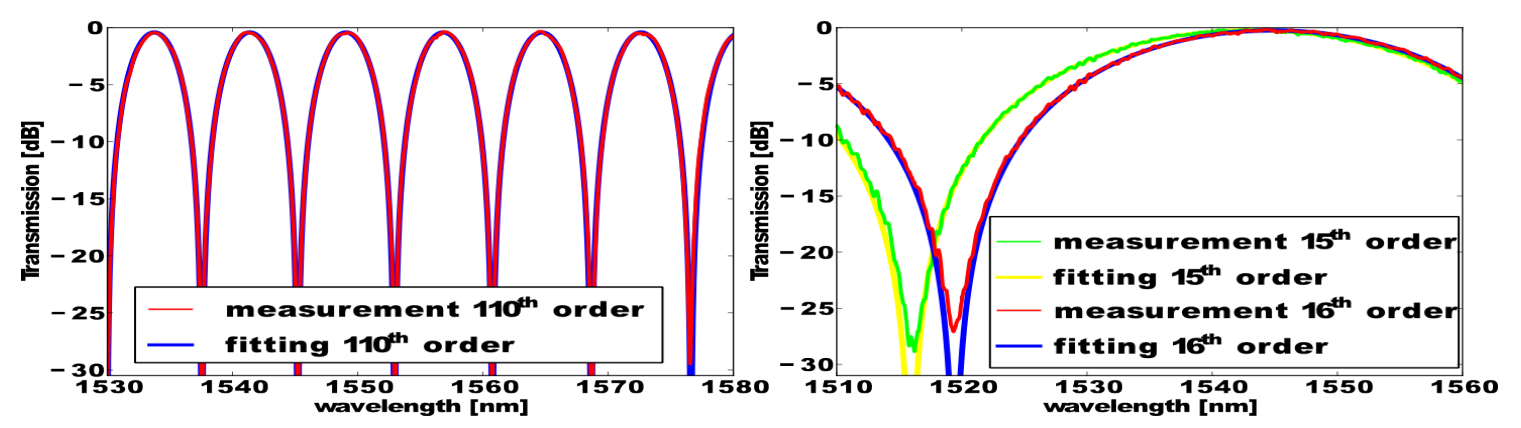

Fig. 2: Measured and fitted normalized spectrum of MZI (a) higher filter order and (b) lower filter order.

\section{Method and Design}

The presented method is generic in nature and should work for all integrated waveguide platforms. In a Mach-Zehnder interferometer, we get constructive interference at the output when

$$
m \cdot \lambda=n_{e f f} . \Delta L
$$

where $\lambda$ is resonant wavelength, $m$ is the filter order, $n_{e f f}$ is effective index of the waveguide at that wavelength and $\Delta L$ is the physical path length difference. The FSR of the filter is the difference between two consecutive peaks of the filter:

$$
F S R=\lambda^{2} / n_{g} . \Delta L
$$

From Eq.1, we designed two MZIs with consecutive order $m$ and $m+1$ in such a way that only one resonant wavelength falls in the entire C-band. For this, we used a simulated model of $n_{\text {eff }}$, making sure that even with a significant deviation from the model we could still identify the peaks of the right order. The third MZI has a much higher order such that we can get number of peaks and valleys in the wavelength band. From this device, measuring $n_{g}$ of the waveguide is relatively straightforward by extracting the wavelength dependent FSR. With this $n_{g}$, we can now extract $n_{e f f}$ based on the exact references of the two low-order MZIs, by modifying the Taylor's expansion

$$
n_{g}(\lambda)=n_{e f f}\left(\lambda_{0}\right)-\lambda_{0} \cdot\left(d n_{e f f} / d \lambda\right)-\lambda_{0} \cdot \Delta \lambda \cdot\left(d^{2} n_{e f f} / d \lambda^{2}\right)
$$

where $\lambda_{0}$ is the center wavelength, in our case its $1550 \mathrm{~nm}$. The further terms of the Taylor's expansion are neglected since they are comparable to the fitting error for the designed TE-polarized waveguide. The eigen mode simulations for the initial estimate of the SOI waveguide mode are performed by the finite element method (FEM) taking a wavelength and temperature dependent material model based on the Sellmeier equation. The MZIs are designed and simulated for $450 \mathrm{~nm}$ wide and $215 \mathrm{~nm}$ thick silicon wires and for values $\mathrm{m}=15,16$ and 110 respectively. Devices are designed using the IPKISS parametric design framework. The device uses compact grating couplers [6] with reduced back reflections for light coupling in and out from the SOI chip. MZI delay lines are designed with a relatively large bend radius of $20 \mu \mathrm{m}$ to minimize the reflections from bends and loss. The actual $\Delta L$ comes only from the straight section of the lower arm of the MZI and not from the bends. this cancels out possible effects of bend-radius dependent $n_{e f f}$. Here we focus on measuring and extracting the $n_{\text {eff }}$ of straight photonic wire. For splitter and combiner we use a $1 \times 2$ multimode interference (MMI). The device was fabricated in the imec $200 \mathrm{~mm}$ CMOS pilot line, using siliconon-insulator wafers with an initial $220 \mathrm{~nm}$ of silicon and $2 \mu \mathrm{m}$ of buried oxide. Camera images of all three MZIs and SEM image of fabricated MZI with $\mathrm{m}=110$ are shown in Fig. 1.

\section{Measurements and Analysis}

The transmission measurement is performed in a thermally controlled environment to eliminate the effect of the high thermo- optic (TO) coefficient of silicon, which can induce a wavelength peak shift about $80 \mathrm{pm} / \mathrm{K}$. The device is first normalized to a simple straight waveguide to eliminate the grating coupler spectrum. The $10 \mathrm{~dB}$ bandwidth of the grating coupler is around $80 \mathrm{~nm}$ from $1505 \mathrm{~nm}$ to $1585 \mathrm{~nm}$. The insertion loss of the devices are less than $0.3 \mathrm{~dB}$. The normalized transmission spectrum is fitted using the non-linear least square method and cosine square interferometric model as a base function. The measured and fitted transmission spectrum of higher and lower are MZIs are shown 

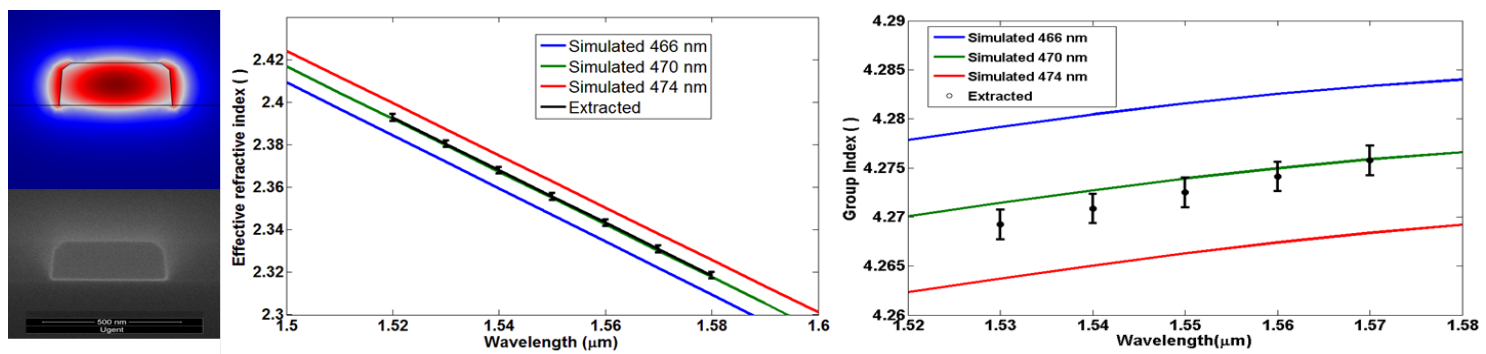

Fig. 3: (a) Simulated mode profile and XSEM image of $470 \mathrm{~nm}$ wide and 211 thick waveguide (b) Simulated and extracted $n_{\text {eff }}$ and (c) Simulated and extracted $n_{g}$ over C-band
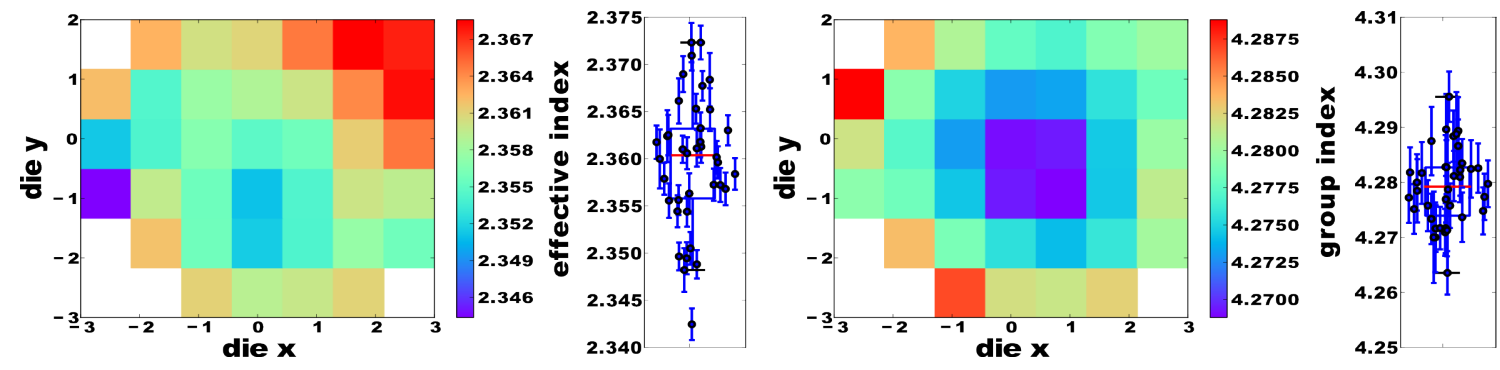

Fig. 4: Wafer statistics and map at $1550 \mathrm{~nm}$ (a) $n_{e f f}$ and (b) $n_{g}$

in Fig. 2.The designed waveguide is $450 \mathrm{~nm}$ wide and $215 \mathrm{~nm}$ thick ( $5 \mathrm{~nm}$ silicon is removed during processing). However, the measured width and thickness of the waveguide is closer to $470 \pm 4 \mathrm{~nm}$ and $211 \pm 1 \mathrm{~nm}$ respectively, as extracted from SEM inspection. The cross-section SEM (XSEM) of the waveguide, the simulated mode profile, the extracted $n_{\text {eff }}$ and $n_{g}$ over the entire C-band is shown in Fig. 3 (a), (b) and (c) respectively. The extracted $n_{g}$ from the higher order filter and then the $n_{\text {eff }}$ from low order filter of the $470 \mathrm{~nm}$ wide and $211 \mathrm{~nm}$ thick waveguide at $1550 \mathrm{~nm}$ is $4.272 \pm 0.0015$ and $2.356 \pm 0.0045$ respectively. The uncertainty is mainly due to fitting and measurements errors. The mismatch between the extracted value and the simulations of the waveguide according to XSEM (with best fitted width, taking into account the uncertaintly of the SEM measurement itself) is less than 0.0007 for $n_{\text {eff }}$ and 0.005 for $n_{g}$ and it mainly comes from local environmental variations, and fabricated waveguide geometrical non-idealities. The wafer statistics and map plot of extracted $n_{e f f}$ and $n_{g}$ are shown in Fig.4 (a), (b). Across the wafer the mean value and standard deviation of $n_{e f f}$ is $2.360 \pm 0.0030$ and 0.007 at $1550 \mathrm{~nm}$ and for $n_{g}$ its $4.279 \pm 0.0040$ and 0.006 respectively.

\section{Conclusion}

We proposed and demonstrated a generic method for measuring the refractive index of TE polarized SOI waveguides.

This method should work for TM polarization and can be extended to other waveguide platforms as well.

\section{References}

1. W. Bogaerts, S. Selvaraja, P. Dumon, J. Brouckaert, K. De Vos, D. Van Thourhout, and R. Baets, "Silicon-on-insulator spectral filters fabricated with cmos technology," Selected Topics in Quantum Electronics, IEEE Journal of 16, 33-44 (2010).

2. J. Jágerská, N. L. Thomas, R. Houdré, J. Bolten, C. Moormann, T. Wahlbrink, J. Ctyroký, M. Waldow, and M. Först, "Dispersion properties of silicon nanophotonic waveguides investigated with fourier optics," Opt. Lett. 32, 2723-2725 (2007).

3. S. Selvaraja, W. Bogaerts, P. Dumon, D. Van Thourhout, and R. Baets, "Subnanometer linewidth uniformity in silicon nanophotonic waveguide devices using cmos fabrication technology," Selected Topics in Quantum Electronics, IEEE Journal of 16, 316-324 (2010).

4. E. Dulkeith, F. Xia, L. Schares, W. M. J. Green, and Y. A. Vlasov, "Group index and group velocity dispersion in silicon-on-insulator photonic wires," Opt. Express 14, 3853-3863 (2006).

5. X. Chen, Z. Li, M. Mohamed, L. Shang, and A. R. Mickelson, "Parameter extraction from fabricated silicon photonic devices," Appl. Opt. 53, 1396-1405 (2014).

6. Y. Li, D. Vermeulen, Y. D. Koninck, G. Yurtsever, G. Roelkens, and R. Baets, "Compact grating couplers on silicon-on-insulator with reduced backreflection,” Opt. Lett. 37, 4356-4358 (2012). 